\title{
Monitoring Methods of Marine Pollution Range Based on Big Data Technology
}

\author{
Q. Zhong*† and X.M. Liu** \\ *Department of Art Design and Public Management, Yantai Vocational College, Yantai 264670, China \\ **Department of Information Engineering, Yantai Vocational College, Yantai 264670, China \\ $†$ Corresponding author: zhongqiang1015@163.com
}

\author{
Nat. Env. \& Poll. Tech. \\ Website: www.neptjournal.com \\ Received: 20-11-2020 \\ Revised: $23-12-2020$ \\ Accepted: 22-01-2021 \\ Key Words: \\ Big data technology \\ Marine pollution \\ Image monitoring \\ Digital remote sensing \\ Intelligent monitoring
}

\section{ABSTRACT}

With the development of big data technology, traditional monitoring methods for the scope of marine pollution can no longer meet the current needs of accuracy and timeliness. In light of the outstanding topic, this study proposed to use big data technology to monitor the scope of marine pollution. The intelligent digital remote sensing technology was used for multi-dimensional monitoring of ocean water quality and completed the calculation of data collected by water quality sensors through the improved big data comparative analysis method. Finally, the scope of pollution monitoring was realized. The results verified that the proposed monitoring method could achieve high-precision and time-sensitive monitoring of the range of marine pollutants, and could identify the basic information of pollutants.

\section{INTRODUCTION}

The ocean and humans have become increasingly intertwined as science and technology have advanced (Cavallaro et al. 2017). And the protection of the marine environment has become a common concern topic for eco-environmental experts. The current common monitoring method for the scope of marine pollution is low-dimensional detection technology, which is characterized by a narrow monitoring range and applies to the near sea area (Sun et al. 2018). At the same time, the monitoring accuracy of this technology is relatively low, and the monitoring time is comparatively long, which may cause the location of pollutants to change during this period (Rabinskiy et al. 2017). Intelligent digital remote sensing technology has been widely used in many industries and has achieved breakthrough achievements as a major application of big data technology in the field of artificial intelligence, but this technology applied to the monitoring of marine pollution is relatively rare. In view of this, this study proposed the use of intelligent digital remote sensing technology and big data comparison technology as a monitoring method for the scope of marine pollution, aiming to provide technical support for future marine environmental management and help to create a green environment.

\section{MATERIALS AND METHODS}

\author{
Marine Pollution Scope Monitoring Method Based on \\ Big Data Technology
}

\section{Intelligent digital remote sensing technology}

Research has introduced intelligent digital remote sensing technology for monitoring the scope of marine pollution. The technology, based on remote sensing satellites, detects marine environmental pollution through intelligent data modules (Verfuss et al. 2018), and then, obtains valuable information through multi-layer database comparison. Intelligent remote sensing technology is different from ordinary remote sensing technology. Its characteristics are more intelligent and digital, which can achieve the effective processing of image information more quickly, allowing users to extract valuable information and apply it to real life and work. Remote sensing technology includes information sources, obtaining information, receiving information, processing information, and applying information (Jauzein et al. 2016). The ocean analysis database uses the object features extracted from the ocean object database to realize data analysis and finally displays it on the computer screen through learning mode, machine learning, data mining, etc. The schematic diagram of the structure of the remote sensing database is shown in Fig. 1. The technical structure includes an image layer, a marine information display layer, and a marine analysis display layer. The image layer is to obtain the information of the marine environment through remote sensing recognition of remote sensing satellites and send the obtained images to the marine information processing interface via the wireless network in the form of a packaged file, which involves image processing, retrieval of object attributes and image characteristics (Setala 
et al. 2016). The marine information processing layer converts monitoring information into digital information through database processing. Marine object databases and marine environmental physical field analysis databases are used. The ocean analysis display layer accepts the transferred data and then processes the image through data link mode, image data module, and data mining technology (Arslan \& Avsar 2020).

\section{Water quality sensor measurement and monitoring technology}

Marine pollutants can usually be divided into petroleum and its corresponding products, metal and non-metal pollutants, acidic or alkaline pollutants, pesticides, radioactive substances, organic pollutants, domestic sewage, thermal pollution, solid waste, antibiotics, etc. Marine polluted waters usually have a variety of pollutants, and the main impacts are reflected in the following three aspects (Bonanno \& Orlando-Bonaca 2018). First, pollutants will cause a series of physical, chemical, and geological reactions that affect the safety of marine organisms. Second, it threatens human life and health through direct channels and the food chain. Third, marine pollutants have undergone major changes over time and in sea conditions. Current monitoring of pollutants includes water quality monitoring, bottom quality monitoring, atmospheric monitoring, biological monitoring, etc.

Based on the above analysis and previous research experience, the research selects water quality sensors to collect marine pollution data and then determines the pollution scope (Ternengo et al. 2018). The monitored data information includes seawater $\mathrm{PH}$ value, seawater dissolved oxygen content, seawater conductivity, and seawater temperature. The types and related functions of the water quality sensors used in the research are shown in Table 1.

\section{Improved big data comparative analysis method}

The research uses a comparative analysis method for the data of marine pollution range. Big data core technologies include four core technologies: big data collection, big data preprocessing, big data storage, and big data analysis. Data preprocessing is directly related to the accuracy of data, and mainly includes four parts: data cleaning, data integration, data conversion, and data specification (Collins \& Halliday 2018). Effective information may be derived from the data using intelligent digital remote sensing technology and

Table 1: Types of water quality sensors and their functions.

\begin{tabular}{|ll|}
\hline Sensor & Main functions \\
\hline PH sensor & Monitoring and measuring the $\mathrm{pH}$ value of seawater \\
Dissolved oxygen sensor & Monitoring and measurement of dissolved oxygen in seawater \\
TriE Conductivity Sensor & The electrical conductivity of seawater was monitored and measured \\
Temperature sensor & The temperature of seawater at different depths is monitored and measured \\
\hline
\end{tabular}

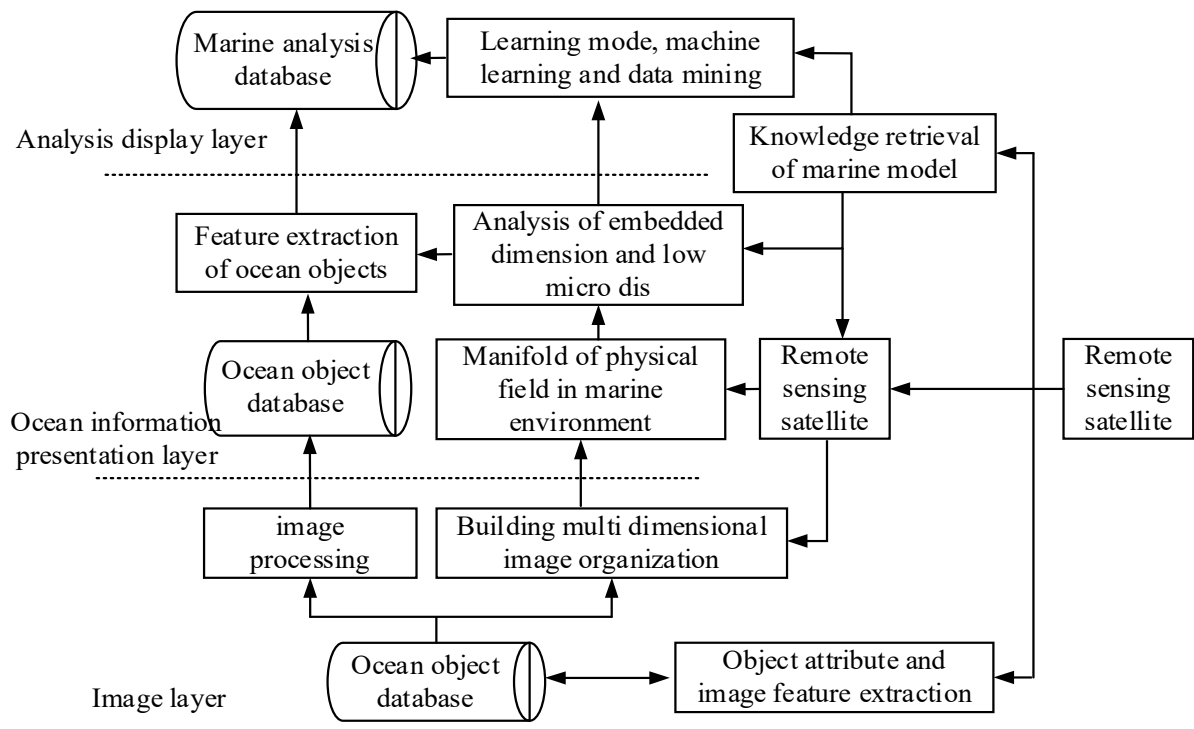

Fig. 1: Structure of intelligent data remote sensing database. 
comprehensive data obtained from water quality sensor monitoring technology to realize pollutant range monitoring (González-Fernández et al. 2016). Normally, the scale of data extraction is huge, which increases the difficulty of comparative analysis. This study uses a spatial comparison method, which is to compare spatial index data within the same time range. The specific implementation steps are as follows. First, redefine the type of data source obtained, then extract the picture information and original data, and perform big data analysis with both marine pollution parameters and standard pollution images to obtain the final comprehensive pollution monitoring result (Alothman et al. 2020).

In the ocean reality, there are often upstream, downstream, sea breeze, and low visibility, so the big data obtained has the characteristics of "reasonable quantification". When the phenomenon of data eddy occurs, the data obtained by the computer will produce analysis and quantitative changes. The law of change is shown in Fig. 2. Fig. 2(a) is a simplified schematic diagram of the vortex degree of the data. The rotation axis of the vortex in the ocean is perpendicular to the earth's surface. The study only considers the motion of the vortex relative to the earth below, that is, the relative vorticity. Fig. 2(b) refers to the relationship between the precise value of the model and the quantitative analysis.

The data eddy current makes the process of analyzing pollution data more difficult, so the study unified quantification of different data disguise, data vector, and data imbalance. When the data eddy current transformation occurs, the data obtained by the computer will also undergo analysis and quantitative changes. The law of change is shown in Fig. 3. Fig. 3(a) is a simplified schematic diagram of the data eddy current under transformation conditions.
The eddy current transformation is to eliminate data interference through certain processing, which is convenient for the accurate analysis of data information. Fig. 3(b) refers to the relationship between the model's precise value and the analytical quantification. After the data vortex changes, the data changes have no effect on the degree, and the big data monitoring parameters and quantified values are corrected on average, considerably reducing the difficulty of big data comparison analysis, resulting in well-realized big data comparative analysis.

\section{RESULTS AND DISCUSSION}

To test the detection effect of the big data technology proposed by the institute in the scope of marine pollutants, the specific information is shown in Table 2. All the pollution sources used in the simulated pollution source experiment are simulated pollution sources to prevent damage to the marine environment (Emeh \& Igwe 2018, Sajil Kumar 2020, Yan et al. 2020). The experiment evaluated the effectiveness of the monitoring method proposed by the institute through the monitoring and identification index. The monitoring identification is the comparison between the number of experiments to find the pollutants and the total number of experiments.

The study selected a fixed $1000 \mathrm{~km}^{2}$ monitoring sea area and simulates the monitoring effects of two technologies, the original ocean monitoring technology and the big data monitoring technology, under different pollution source areas. Following the recording of the monitoring results, the pollution monitoring experiment was conducted under the conditions of modifying the sea area and selecting different flow rates and contrasts, and the monitoring data was recorded. The obtained relationship between the degree of

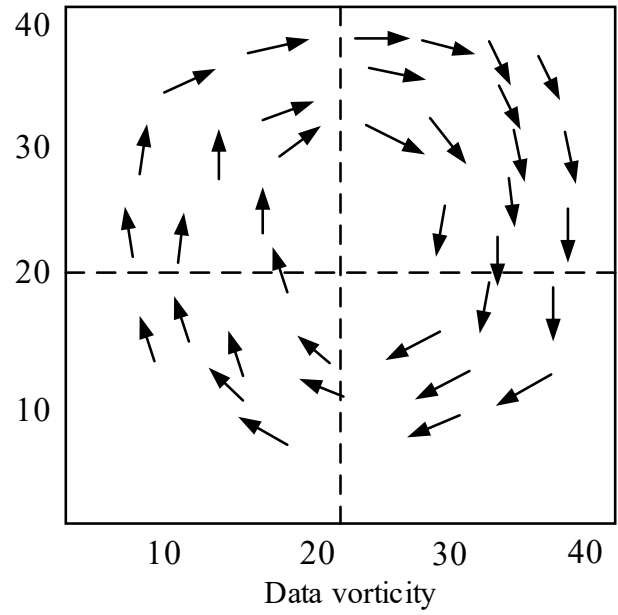

(a)



(b)

Fig. 2: Analysis and quantification change rule under data eddy current. 
identification and the simulated pollution source is shown in Fig. 4. White and black respectively indicate the degree of identification of the original marine pollution monitoring technology and intelligent image monitoring technology. When the area of the monitored pollution source is $200 \mathrm{~m}^{2}$ and $300 \mathrm{~m}^{2}$, the corresponding monitoring recognition degree is lower than $80 \%$ and $85 \%$ respectively. When the pollutant area drops again, the data obtained will not be of reference. The intelligent image monitoring method based on big data technology proposed by the research institute can achieve a recognition rate of about $92 \%$ even when the pollution area is $72 \mathrm{~m}^{2}$, and the monitoring recognition rate stabilizes at about $95 \%$ as the pollution area increases.

The experiment further verified the timeliness of the intelligent image monitoring method based on big data technology. Select a certain fixed sea area and compare the monitoring time of $100 \mathrm{~km}^{2}$ simulated pollution source through two monitoring methods. Record the monitoring results and draw the curve diagram of the detection time and pollution source as shown in Fig. 5. It can be seen from Fig. 5 that when the pollution source range is small, the monitoring time of the two monitoring methods differs greatly. Traditional monitoring methods have a relatively long monitoring time when the pollution range is relatively small. In this monitoring method, when the pollution range is within $300 \mathrm{~m}^{2}$, the monitoring time is $10 \mathrm{~min}$, and when the monitoring range is within $150 \mathrm{~m}^{2}$, the monitoring time is $15 \mathrm{~min}$. The monitoring method based on big data technology has very high monitoring timeliness even under a pollution area of about $50 \mathrm{~m}^{2}$. It only takes 5 minutes, and the monitoring time is usually stable at about 5 minutes.

\section{CONCLUSION}

Aiming at the current widespread marine pollution problem, this research proposes an intelligent digital image monitoring method for marine pollutants based on big data technology, using a variety of water quality sensors to collect data, and using intelligent digital image sensing technology for image processing and analysis. Finally, the optimized big data comparative analysis method is used for big data analysis, and the final result is obtained. Through the detection of marine pollutant detection range, identification degree detection, and timeliness detection, the results verify that the proposed intelligent digital detection method based on big data technology can achieve good pollutant monitoring. Under the condition of the low pollution area, the recognition degree

Table 2: Experimental data information.

\begin{tabular}{|llll|}
\hline Simulation pollution source $/ \mathrm{m}^{2}$ & Monitoring area $/ \mathrm{km}^{2}$ & Simulation pollution source $/ \mathrm{m}^{2}$ & ${\mathrm{Monitoring} \mathrm{area} / \mathrm{km}^{2}}^{2000}$ \\
\hline 50 & 500 & 200 & 2250 \\
75 & 750 & 225 & 2500 \\
100 & 1000 & 250 & 2750 \\
125 & 1250 & 275 & 3000 \\
150 & 1500 & 300 & 325 \\
175 & 1750 & & 3250 \\
\hline
\end{tabular}

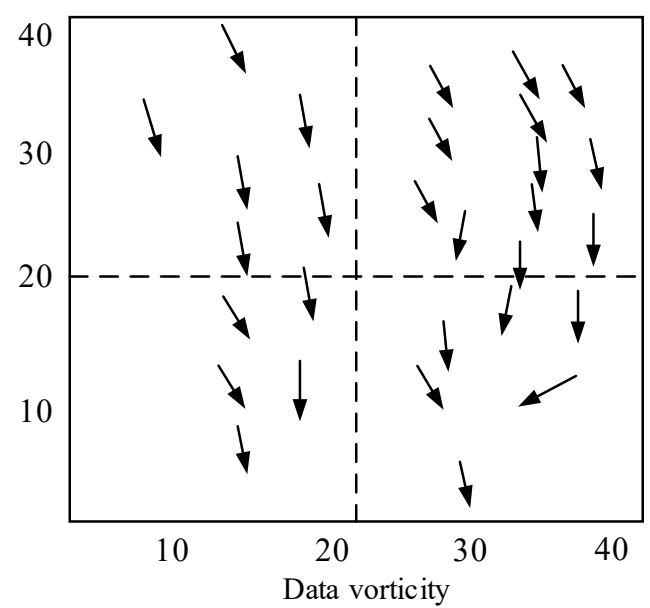

(a)

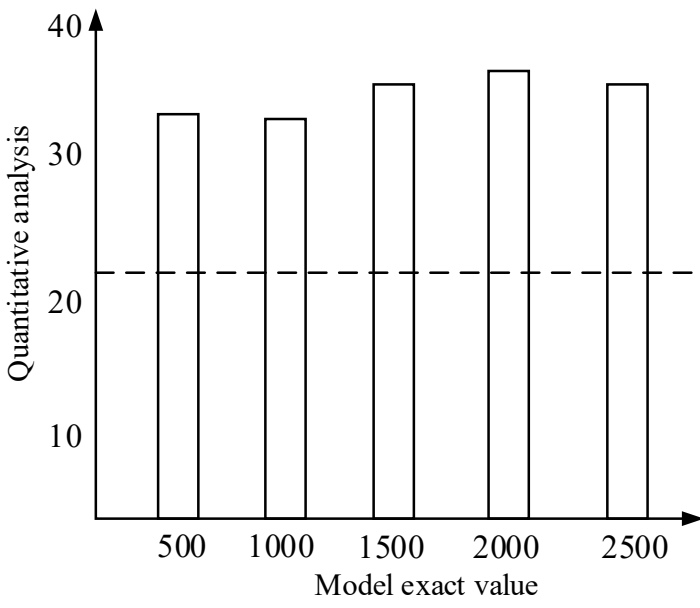

(b)

Fig. 3: Analysis and quantification change rule of data eddy current transformation. 


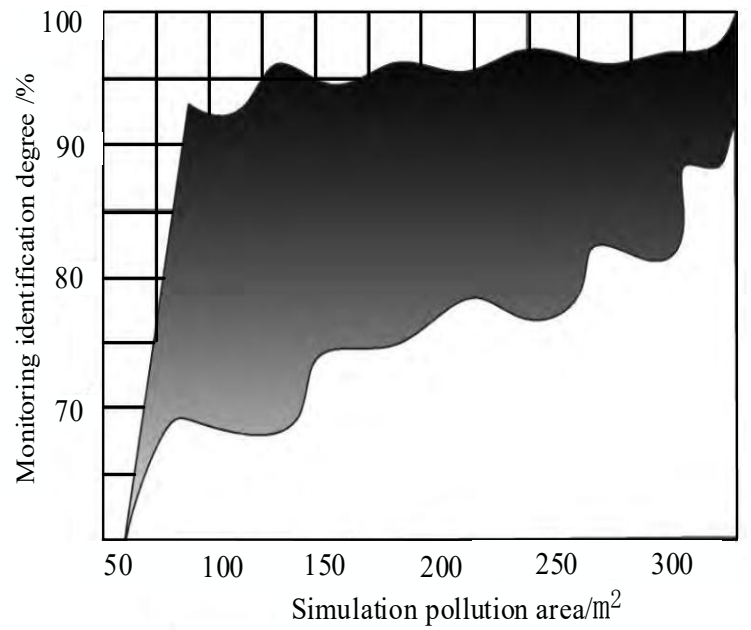

Fig. 4: Simulation pollution source area and monitoring is the rate curve.



Fig. 5: Simulation pollution source and monitoring time curve.

of the intelligent image monitoring method is about $92 \%$. As the pollution area increases, the recognition degree of monitoring stabilizes to $95 \%$. And the monitoring timeliness of this method is also very high, and the monitoring time is usually stable at 5 minutes. When the original detection method monitors the pollution source area of $200 \mathrm{~m}^{2}$ and $300 \mathrm{~m}^{2}$, the monitoring recognition rate is as low as $80 \%$ and $85 \%$, respectively. At the same time, when the pollution range is within $300 \mathrm{~m}^{2}$ and $150 \mathrm{~m}^{2}$, the monitoring time is as high as $10 \mathrm{~min}$ and $15 \mathrm{~min}$.

\section{REFERENCES}

Alothman, Z.A., Habila, M.A. and Moshab, M.S. 2020. Fabrication of renewable palm-pruning leaves-based nano-composite for remediation of heavy metals pollution. Arab. J. Chem., 13(4): 4936-4944.
Arslan, S. and Avsar, O. 2020. Assessment of heavy metal pollution in Koycegiz-Dalyan coastal lagoon watershed (Mugla) SW Turkey. Arab. J. Geosci., 13(71915)

Bonanno, G. and Orlando-Bonaca, M. 2018. Perspectives on Using Marine Species as Bioindicators of Plastic Pollution. Mar. Pollut. Bull., 137C (DEC.): 209-221.

Cavallaro, G., Riedel, M. and Richerzhagen, M. 2017. On understanding big data impacts in remotely sensed image classification using support vector machine methods. IEEE J. Sel. Top. Appl. Earth Obs. Remote Sens., 8(10): 4634-4646.

Collins, J.P. and Halliday T. 2005. Forecasting changes in empirical biology: Aiming at a moving target. Philos. Trans. R. Soc. Lond., B, Biol. Sci., 360(1454): 309-14

Emeh, C. and Igwe, O. 2018. Effect of environmental pollution on the susceptibility of sesquioxide-rich soils to water erosion. Geol. Ecol. Landscapes, 2(2): 115-126.

González-Fernández, C., Albentosa, M. and Campillo, J.A.. 2016. Effect of mussel reproductive status on biomarker responses to PAHs: Implications for large-scale monitoring programs. Aquat. Toxicol., 177: 380-394. 
Jauzein, C., Fricke, A. and Mangialajo, L. 2016. A sampling of Ostreopsis cf. ovata using artificial substrates: optimization of methods for the monitoring of benthic harmful algal blooms. Mar. Pollut. Bull., 107(1): 300-304.

Rabinskiy, L.N., Ripetskiy, A.V. and Zelenov, S.V. 2017. Analysis and monitoring methods of technological preparation of additive production. J. Ind. Pollut. Control., 33(1): 1178-1183.

Sajil Kumar, P. J. 2020. Hydrogeochemical and multivariate statistical appraisal of pollution sources in the groundwater of the lower Bhavani river basin in Tamil Nadu. Geol. Ecol. Landscapes, 4(1): 40-51.

Setala, O., Magnusson, K. and Lehtiniemi, M. 2016. Distribution and abundance of surface water microlitter in the Baltic Sea: A comparison of two sampling methods. Mar. Pollut. Bull., 110(1): 177-183.
Sun, X., Tian, Z. and Malekian, R. 2018. Estimation of vessel emissions inventory in Qingdao Port based on big data analysis. Symmetry, 10(10): 452-452.

Ternengo, S., Marengo, M. and El Idrissi, O. 2018. Spatial variations in trace element concentrations of the sea urchin, Paracentrotus lividus: A first reference study in the Mediterranean Sea. Mar. Pollut. Bull., 129 (APR.): 293-298.

Verfuss, U.K, Gillespie, D. and Gordon, J. 2018. Comparing methods suitable for monitoring marine mammals in low visibility conditions during seismic surveys. Mar. Pollut. Bull., 126(1): 1-18.

Yan, Y., Sun, L. and Peng, Z. 2020. Effects of pyrolyzed semi-char blend ratio on coal combustion and pollution emission in a $0.35 \mathrm{MW}$ pulverized coal-fired furnace. Front. Energy, 5(6): 71-89. 